\title{
Secondary infarction in single or in multiple vascular territories: two different entities following subarachnoid hemorrhage?
}

\author{
N. K. de Rooij • C. J. M. Frijns • B. K. Velthuis • \\ G. J. E. Rinkel
}

Received: 28 January 2011/Revised: 14 April 2011/Accepted: 19 April 2011 / Published online: 7 May 2011

(C) The Author(s) 2011. This article is published with open access at Springerlink.com

\begin{abstract}
The pathogenesis of secondary infarctions (SI) after aneurysmal subarachnoid hemorrhage (SAH) is poorly understood. To assess whether SI in single (SSI) or multiple (MSI) vascular territories represent different disease entities, we compared clinical profiles of patients with these patterns of SI. CT/MRI-examinations of 448 patients were reviewed for new infarctions within 28 days after $\mathrm{SAH}$, and categorized into SSI or MSI. Only patients with adequate follow-up imaging excluding any new infarctions were included for analysis (269 patients). Procedure-related infarctions were excluded. Odds ratios (ORs) with corresponding 95\% confidence intervals (CI) were calculated for patients with SSI or MSI versus patients without SI to analyze differences in demographic characteristics, vascular risk factors, disease-related characteristics and treatment modalities. Thirty-six patients had SSI, 53 MSI and 180 no SI. ORs in MSI-patients were $>1.5$ times higher compared with ORs in SSI-patients for multiple vascular risk factors [MSI:5.4 (2.3-13) versus SSI:1.2 (0.5-2.8)], poor clinical condition on admission [MSI:4.6 (2.4-8.9) versus SSI:2.4 (1.1-5.2)], initial loss of consciousness [MSI:2.6 (1.3-5.3) versus SSI:1.1 (0.5-2.3)] and large
\end{abstract}

N. K. de Rooij - C. J. M. Frijns · G. J. E. Rinkel Utrecht Stroke Center, Department of Neurology and Neurosurgery, Rudolf Magnus Institute of Neuroscience, University Medical Center Utrecht, Utrecht, The Netherlands

B. K. Velthuis

Department of Radiology, University Medical Center Utrecht, Utrecht, The Netherlands

N. K. de Rooij ( $\square)$

Department of Neurology, University Medical Center Utrecht, Room H02.128, PO Box 85500, 3508 GA Utrecht,

The Netherlands

e-mail: n.k.derooij@umcutrecht.nl amounts of intraventricular blood [MSI:2.9 (1.4-5.8) versus SSI:1.5 (0.7-3.2)]. In multivariate analysis ORs remained higher in MSI for presence of multiple vascular risk factors [MSI:1.9 (1.2-2.9) versus SSI:1.1 (0.8-1.7)] and initial loss of consciousness [MSI:3.0 (1.0-8.9) versus SSI:1.6 (0.6-4.0)]. Our findings suggest that SSI and MSI after SAH are not distinct disease entities. MSI was related to the same characteristics as SSI but to a larger extent, specifically to the presence of multiple vascular risk factors, initial loss of consciousness, larger amounts of intraventricular blood, and poor clinical status on admission.

Keywords Delayed cerebral ischemia - Secondary infarction - Subarachnoid hemorrhage

\section{Introduction}

Delayed cerebral ischemia (DCI) is a major cause of poor outcome after subarachnoid hemorrhage (SAH). Although cerebral vasospasm has been indicated as the main cause of DCI [13], vasospasm and DCI do not always go hand in hand, which shows that vasospasm is not the only or essential factor for the development of DCI [2, 3, 9]. Other factors that may contribute to DCI besides large vessel vasospasm are inflammatory processes, intracranial microthrombosis, cortical spreading depolarisations and impaired cerebral autoregulation $[4,8,14]$.

DCI may have several patterns. From a subdivision based on number and sites of infarctions on cerebral computed tomographic (CT) scans after SAH, two common patterns were distinguished [10]. These two common patterns were on the one hand a single cortical infarct and on the other multiple cortical and/or deep cerebral infarcts. It is unclear whether these secondary infarct (SI) patterns 
represent pathophysiologically different disease entities or different degrees of severity of the same vascular process.

We assessed whether secondary infarctions in single (SSI) or multiple (MSI) vascular territories represent different disease entities by comparing the clinical profiles of the patients with SSI or MSI with those of patients without infarction. We analyzed demographic characteristics, vascular risk factors, disease specific characteristics, and treatment modalities as determinants for development of SI.

\section{Methods}

Patients and data

From the prospectively collected database of all consecutive patients with SAH admitted to the University Medical Center Utrecht, we included all patients with an aneurysmal SAH who were admitted within 3 days after onset between January 1999 and June 2007. The diagnosis of SAH was based on a positive CT scan or xantochromia of the cerebrospinal fluid. Presence of an aneurysm had to be confirmed by means of CT, MR or catheter angiography. For inclusion in the current study at least one follow-up scan, performed more than $24 \mathrm{~h}$ after the initial CT scan, was required.

For every patient we recorded data on gender, age, current smoking, history of hypertension, previous cardiovascular events (including stroke, myocardial infarction and peripheral vascular disease), hypercholesterolemia, diabetes mellitus, family history of cardiovascular events, and use of salicylic acid and statins at the time of admission. The clinical condition on admission was assessed with the World Federation of Neurological Surgeons scale and dichotomized in good (WFNS I to III) or poor (IV or V) [1]. We also recorded loss of consciousness at onset, and modality and timing of intervention. All patients were treated according to our standard protocol, including absolute bed rest, compressive stockings, intensive monitoring on our medium or intensive care unit until aneurysm treatment, nimodipine $360 \mathrm{mg}$ daily orally, and intravenous administration of fluids aiming at normovolemia for 3 weeks.

Initial CT scans were evaluated for amount of blood, hydrocephalus and pre-existing infarction. The amount of cisternal and ventricular blood was assessed according to the method described by Hijdra et al. [7] and dichotomized at their median values. The presence of acute hydrocephalus was quantified and adjusted for age by means of the bicaudate index (BCI) divided by the corresponding upper limit per age group [6]. Acute hydrocephalus was defined as an age-adjusted relative BCI of $>1$.
Follow-up scans were evaluated for new ischemic lesions. Lesions caused by extraventricular drains, preexisting infarcts and hypodensities around a hematoma or in the vicinity of the operation area were not considered new infarctions. Only infarctions unrelated to angiography or aneurysm treatment were defined as "spontaneous infarction". Infarctions were considered to be related to treatment if new neurological symptoms occurred directly after aneurysm treatment, and an infarct was visible in the territory of the parent vessel of the aneurysm on CT within $48 \mathrm{~h}$ after treatment. In case the time of development of infarction was uncertain (for example if a new infarction was present on $\mathrm{CT}>48 \mathrm{~h}$ after treatment and/or the neurological state of the patient directly after treatment could not be assessed due to sedative medication), an expert opinion was made by two of the authors, who were not aware of the risk factor profile. In this expert opinion the report of the operation or endovascular treatment was taken into account. In case of disagreement, the infarction was not counted as spontaneous infarction. To investigate if differentiation between edema and infarction could reliably be made in the presence of an intracerebral hematoma (ICH), we performed an interobserver study between three observers (two senior neurologists and one senior radiologist) in 25 patients with an additional ICH and a surrounding hypodensity. This study showed a large interobserver variability with kappa values around 0.50 (95\%CI 0.06-0.96). Since subdivision in SSI and MSI in these cases could not reliably be made, patients with an ICH and a surrounding hypodensity were excluded.

The location(s) of spontaneous infarctions were determined by using validated arterial territory maps [11, 12]. We only included infarctions that developed within 28 days after SAH and were confirmed on a late follow-up scan. SSI was defined as infarction in one arterial territory and MSI as infarctions in more than one territory. Adequate follow-up (late CT or MRI follow-up between 28 days and 6 months after SAH) is necessary because within the first 28 days SSI could develop into MSI, and NSI could develop into SSI or MSI. Because new (silent) infarctions in other territories had to be excluded for a valid subdivision, we excluded patients with a single infarction or without any infarction who had no late follow-up scan.

All scans were reviewed for presence or absence of hypodensities by one author (NKdR). The first 35 scans were also reviewed independently by a senior vascular neurologist (CJMF). No disagreement was noticed with respect to presence or absence of hypodensities. All scans with hypodensities were independently reviewed and subdivided into SSI and MSI by NKdR and CJMF. In case of disagreement, consensus was reached in a meeting of all authors, including another senior vascular neurologist and a senior radiologist. 
Data analysis

All patients with adequate radiological follow-up (SSI, MSI and NSI) were included for analysis. Odds ratios (ORs) for MSI or SSI compared with NSI were calculated with corresponding 95\% confidence intervals (CI) using univariate logistic regression. We decided to perform an indirect comparison between MSI and SSI as primary analysis to increase the statistical power of the study. Furthermore, an indirect comparison reveals both the relative differences in ORs between MSI and SSI, and the absolute ORs for MSI and SSI separately. Additionally, we performed a direct comparison. All patient specific risk factors and disease specific characteristics were analyzed, except for the risk factors found in less than $10 \%$ of the patients. The total number of the vascular risk factors was analyzed as well: age above the median value, male gender, hypertension, previous cardiovascular disease, positive family history of vascular disease and current smoking. We defined high number of vascular risk factors as any number above the median number. Multivariate logistic regression was performed with all variables of which the association with MSI was more than 1.5 times higher or lower than the association with SSI.

\section{Results}

Of 828 consecutive patients with aneurysmal SAH admitted to our hospital within 3 days after onset of the hemorrhage during the study period, we excluded 222 patients with incomplete scans. In 182 of these 222 patients no follow-up scan was made because of good outcome $(n=65)$, transfer to another hospital $(n=7)$, or early death $(n=110)$, and in 40 patients the initial or follow-up CT scan could not be retrieved. We excluded 149 patients because a large ICH with a surrounding hypodensity was present and subdivision in SSI and MSI could not reliably be made. Nine patients, in whom no consensus for presence or timing of infarction could be reached, were also excluded. An infarction related to treatment was recorded in 76 patients (17\%). Eighty-nine of the 269 included patients with adequate follow-up developed spontaneous infarctions: 36 patients in a single vascular territory and 53 patients in multiple territories. In 180 patients no infarction was present (Fig. 1).

Baseline characteristics of these 269 patients are presented in Table 1. The median number of vascular risk factors was two. History of hypercholesterolemia, diabetes mellitus, use of salicylic acid and use of statins were recorded in less than $10 \%$ of patients, and were not used in the analyses.

Results of the indirect comparison (patients with MSI or SSI vs. NSI) are shown in Table 2. ORs were $\geq 1.5$ for both MSI and SSI in five characteristics: positive family history of cardiovascular events, smoking, poor WFNS, and high amount of both cisternal and ventricular blood. The association with MSI was more than 1.5 times stronger than with SSI in eight characteristics: male gender, high age, history of cardiovascular events,
Fig. 1 Selection of patients with spontaneous single and multiple secondary infarction (SI) within 28 days after subarachnoid hemorrhage (SAH). *All treatment related infarctions were excluded. Depending on the presence or absence of other infarctions these patients were included in the SSI, MSI or the NSI group. ${ }^{\dagger}$ All gray marked patients had adequate radiological follow-up (late follow-up scan between 28 days and 6 months after onset of the $\mathrm{SAH}$ ) and were included for analyses. SSI and NSI were defined as probable if there was no late CT or MRI follow-up to exclude new infarctions in other territories

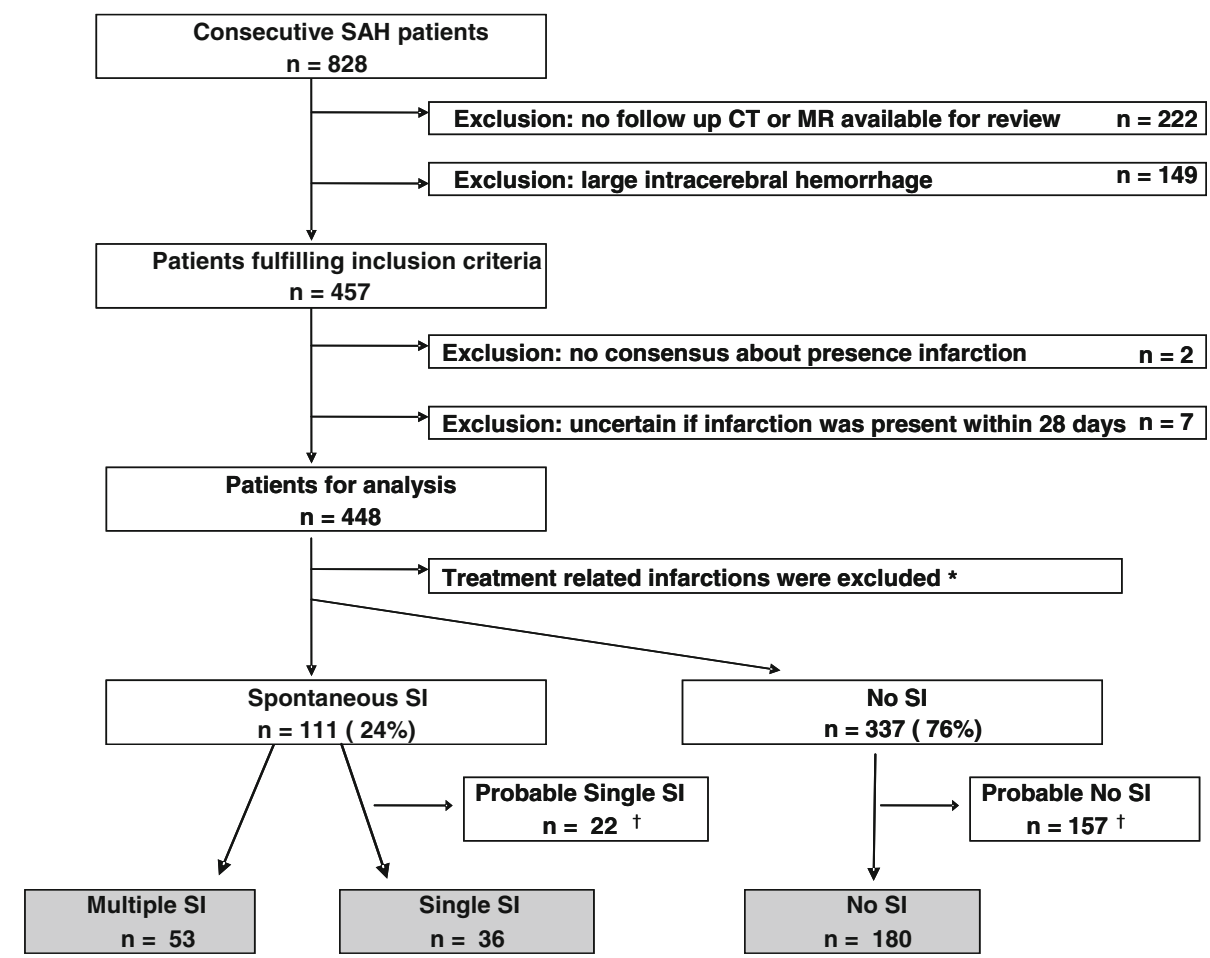


Table 1 Baseline characteristics of 269 included patients

\begin{tabular}{|c|c|c|c|c|}
\hline & $\begin{array}{l}\text { Number of } \\
\text { patients }^{\mathrm{a}}\end{array}$ & $\begin{array}{l}\text { No secondary } \\
\text { infarction }(N=180)\end{array}$ & $\begin{array}{l}\text { Single secondary } \\
\text { infarction }(N=36)\end{array}$ & $\begin{array}{l}\text { Multiple secondary } \\
\text { infarction }(N=53)\end{array}$ \\
\hline \multicolumn{5}{|l|}{ Patient characteristics } \\
\hline Women $(\%)$ & 269 & $129(72)$ & $26(72)$ & $33(62)$ \\
\hline Median age in years (range) & 269 & $54(18-83)$ & $51(26-76)$ & $54(29-77)$ \\
\hline Hypertension $(\%)$ & 263 & $43(24)$ & $8(30)$ & $14(29)$ \\
\hline History of cardiovascular events (\%) & 264 & $26(15)$ & $3(9)$ & $8(16)$ \\
\hline Family history of cardiovascular disease $(\%)^{\mathrm{b}}$ & 269 & $26(14)$ & $9(25)$ & $9(17)$ \\
\hline Diabetes $(\%)$ & 263 & $9(5)$ & $0(0)$ & $3(6)$ \\
\hline Hypercholesterolemia $(\%)^{\mathrm{b}}$ & 269 & $7(4)$ & $3(8)$ & $3(6)$ \\
\hline Current smoking $(\%)$ & 204 & $80(54)$ & $20(67)$ & $18(67)$ \\
\hline Use of salicylic acid (\%) & 247 & $12(7)$ & $5(15)$ & $6(14)$ \\
\hline Use of statins $(\%)$ & 259 & $13(7)$ & $3(8)$ & $4(9)$ \\
\hline \multicolumn{5}{|l|}{ SAH characteristics } \\
\hline Poor WFNS (IV, V) on admission (\%) & 268 & $37(21)$ & $14(39)$ & $29(55)$ \\
\hline Any loss of consciousness at onset $(\%)$ & 239 & $61(37)$ & $12(39)$ & $27(61)$ \\
\hline \multicolumn{5}{|l|}{ Median amount of blood on initial CT } \\
\hline Cisternal blood (range 0-30) & 163 & $24(0-30)$ & $26(0-30)$ & $26(0-30)$ \\
\hline Intraventricular blood (0-12) & 141 & $2(0-12)$ & $2(0-8)$ & $3.5(0-12)$ \\
\hline Acute hydrocephalus ${ }^{c}(\%)$ & & $49(41)$ & $15(50)$ & $22(50)$ \\
\hline External ventricular drainage & 261 & $56(33)$ & $16(44)$ & $19(36)$ \\
\hline Site of the aneurysm (\%) & 269 & & & \\
\hline Posterior communicating artery & & $35(19)$ & $10(28)$ & $13(25)$ \\
\hline Internal carotid artery & & $6(3)$ & $1(3)$ & $5(10)$ \\
\hline Middle cerebral artery & & $23(13)$ & $9(25)$ & $4(8)$ \\
\hline Anterior communicating artery & & $77(43)$ & $9(25)$ & $20(38)$ \\
\hline Pericallosal artery & & $9(5)$ & $0(0)$ & $1(2)$ \\
\hline Basilar artery & & $13(7)$ & $3(8)$ & $2(4)$ \\
\hline Vertebral artery & & $12(7)$ & $1(3)$ & $4(8)$ \\
\hline Other & & $5(3)$ & $3(8)$ & $4(8)$ \\
\hline Treatment of the aneurysm $(\%)$ & 269 & & & \\
\hline No treatment & 23 & $4(2)$ & $0(0)$ & $19(36)$ \\
\hline Coiling & 108 & $75(42)$ & $16(44)$ & $17(32)$ \\
\hline Clipping & 138 & $101(56)$ & $20(56)$ & $17(32)$ \\
\hline Poor Outcome (GOS 1 or 2 ) at discharge (\%) & 256 & $2(1)$ & $2(6)$ & $28(60)$ \\
\hline
\end{tabular}

WFNS World Federation of Neurological Surgeons Scale, GOS Glasgow Outcome Scale

${ }^{a}$ Number of patients is $<269$ in case of unknown data

b These risk factors were recorded as present if they were mentioned as present in patient's history, otherwise as absent or unknown

c Acute hydrocephalus was defined as an age-adjusted relative BCI of $>1$

presence of $>2$ vascular risk factors (including these former three variables), poor WFNS, initial loss of consciousness and high amount of ventricular blood. There were no factors of which the association with SSI was more than 1.5 times stronger than with MSI. In the multivariate analysis the association with MSI remained statistically significant for initial loss of consciousness (OR 3.0; 95\% CI 1.0-8.9) and presence of $>2$ vascular risk factors (OR 1.9; 1.2-2.9) (Table 3).
In the direct comparison (MSI vs. SSI) we found essentially the same associations, however with wider 95\% CIs because of the smaller numbers of patients. Two associations reached statistical significance in the univariate analysis: presence of $>2$ vascular risk factors (OR 4.7; 1.5-14) and any loss of consciousness at onset (OR 2.5; 1.0-6.5). In the multivariate analysis of the direct comparison the presence of $>2$ vascular risk factors remained significantly associated with MSI (OR 1.8; 1.0-3.1). 
Table 2 Differences in clinical and radiological data between patients with single and multiple secondary infarctions (SI) compared with patients without SI

\begin{tabular}{lll}
$\begin{array}{l}\text { Number of } \\
\text { patients }\end{array}$ & $\begin{array}{l}\text { OR }(95 \% \text { CI }) \text { single SI } \\
(n=36) \text { vs. no SI }(n=180)\end{array}$ & $\begin{array}{l}\text { OR }(95 \% \text { CI }) \text { multiple SI } \\
(n=53) \text { vs. no SI }(n=180)\end{array}$ \\
\hline
\end{tabular}

Patient characteristics

\begin{tabular}{|c|c|c|c|}
\hline Male gender & 269 & $1.0(0.4-2.2)$ & $1.5(0.8-2.9)$ \\
\hline Age $\geq 54$ years (median) & 269 & $0.5(0.2-1.1)$ & $1.0(0.6-1.9)$ \\
\hline History of cardiovascular disease & 264 & $0.5(0.2-1.9)$ & $1.1(0.5-2.7)$ \\
\hline History of hypertension & 263 & $0.9(0.4-2.2)$ & $1.3(0.6-2.6)$ \\
\hline Family history of cardiovascular events & 269 & $2.0(0.8-4.7)$ & $1.5(0.9-2.5)$ \\
\hline Current Smoking & 204 & $1.7(0.7-3.8)$ & $1.7(0.7-4.0)$ \\
\hline More than two vascular risk factors ${ }^{a, b}$ & 203 & $1.2(0.5-2.8)$ & $5.4(2.3-13)$ \\
\hline \multicolumn{4}{|l|}{ SAH characteristics } \\
\hline Poor WFNS (IV or V) ${ }^{\mathrm{b}}$ & 268 & $2.4(1.1-5.2)$ & $4.6(2.4-8.9)$ \\
\hline Any loss of consciousness at onset ${ }^{\mathrm{b}}$ & 239 & $1.1(0.5-2.3)$ & $2.6(1.3-5.3)$ \\
\hline Amount of cisternal blood $\geq 25$ (median) & 224 & $2.1(1.0-4.5)$ & $1.5(0.8-2.9)$ \\
\hline Amount of ventricular blood $\geq 2$ (median) ${ }^{b}$ & 219 & $1.5(0.7-3.2)$ & $2.9(1.4-5.8)$ \\
\hline Acute hydrocephalus & 194 & $1.4(0.6-3.2)$ & $1.4(0.7-2.9)$ \\
\hline External ventricular drainage & 261 & $1.7(0.8-3.4)$ & $1.2(0.6-2.2)$ \\
\hline Clipping vs. coiling of the aneurysm & 246 & $0.9(0.5-1.9)$ & $0.7(0.4-1.6)$ \\
\hline
\end{tabular}

${ }^{a}$ Number of following six risk factors: age above the median value, male gender, hypertension, previous cardiovascular disease, positive family history of vascular disease and current smoking. High number of vascular risk factors was defined as any number above the median (two)

${ }^{\mathrm{b}}$ Factors in multivariate analyses

WFNS World Federation of Neurological Surgeons Scale

Table 3 Multivariate analysis of associations of patients' and SAH characteristics with single or multiple secondary infarctions (SI) compared with patients without SI

\begin{tabular}{llll}
\hline & $\begin{array}{l}\text { Number of } \\
\text { patients }\end{array}$ & $\begin{array}{l}\text { OR (95\% CI) single SI } \\
(n=36) \text { vs. no SI }(n=180)\end{array}$ & $\begin{array}{l}\text { OR (95\% CI) multiple SI } \\
(n=53) \text { vs. no SI }(n=180)\end{array}$ \\
\hline More than two vascular risk factors ${ }^{\mathrm{a}}$ & 203 & $1.1(0.8-1.7)$ & $1.9(1.2-2.9)$ \\
Poor WFNS (IV or V) & 268 & $2.1(0.8-5.6)$ & $1.8(0.5-5.9)$ \\
Any loss of consciousness at onset & 239 & $1.6(0.6-4.0)$ & $3.0(1.0-8.9)$ \\
Amount of ventricular blood $\geq 2$ (median) & 219 & $1.0(0.8-1.2)$ & $1.1(0.9-1.3)$ \\
\hline
\end{tabular}

WFNS World Federation of Neurological Surgeons Scale

${ }^{a}$ Number of the following six risk factors: age above the median value, male gender, hypertension, previous cardiovascular disease, positive family history of vascular disease and current smoking. High number of vascular risk factors was defined as any number above the median

\section{Discussion}

Our study showed that all patients with secondary infarction had a similar clinical profile. Both infarct patterns (single or multiple vascular territories) were related to characteristics representing poor initial condition after SAH and presence of vascular risk factors when compared with patients without infarction. However, patients with MSI had an obviously stronger association with these risk factors than patients with SSI, with odds ratios being higher for MSI than for SSI regarding the presence of multiple vascular risk factors, poor neurological condition on admission, initial loss of consciousness, and large amounts of intraventricular blood. These results suggest that SSI and MSI after SAH are not distinct disease entities, but consequences of increasing severity of the same risk factors, and therefore different degrees of the same pathophysiological process.

A previous study distinguished five distribution patterns of infarctions after SAH (based on location and number of infarctions) and analyzed factors that determined their occurrence. The authors suggested that single cortical or multiple cortical and/or deep infarctions are two common types of SI, and may represent different pathophysiological 
mechanisms [10]. That conclusion was based on associations of occurrence of multiple infarctions with history of diabetes, early hydrocephalus and requirement of external ventricular drainage (univariate analysis). Requirement of external ventricular drainage was the only independent predictor in their multivariate analysis. In contrast to our methods, this previous study investigated a small population of 56 patients, directly comparing patients with SI in single or multiple vascular territories, without excluding treatment related infarctions and with limited CT follow-up time. Trends found for associations of multiple infarctions with higher age, poor clinical condition on admission and presence of vascular risk factors corresponded with our findings. Because of the limited statistical power with 56 patients, relevant factors such as initial WFNS score may have been undetected. To reduce this risk of type II error, we made an indirect comparison that included 269 patients. In our study acute hydrocephalus was assessed as BCI adjusted for age, which we feel is a more accurate measure than BCI alone, and found no strong association between MSI and hydrocephalus. The factors that proved to be the most important determinants in our study (presence of initial loss of consciousness and presence of multiple vascular risk factors) were not studied in the previous study.

We studied a large population of 269 SAH patients, 89 of whom developed spontaneous infarctions after SAH. One of the strengths of our study is the meticulous elimination of possible sources of confounding data due to equivocal assessment of spontaneous SI. All CT scans were independently reviewed by two authors and a specialist team in case of disagreement. We performed an interobserver study of the assessment of hypodense lesions at the location of intracerebral hematomas. Because distinction between perihematomal edema and (hemorrhagic) infarction proved difficult to make, we excluded patients with ICH and surrounding hypodense lesions in the comparison between patients with SSI and MSI. For similar reasons we also excluded patients with hypodensities in the operation field. In a study on pathogenesis of DCI, it is essential to include only infarctions of which it is completely sure that they are unrelated to a procedure. For that reason we considered infarction as procedure related, if there was a chance, not only if it was proven to be procedure related. For analysis we included only patients with adequate radiological follow-up. The disadvantage of this elimination of several sources of bias is that we had to exclude large numbers of patients. However, because the original sample we studied was very large, we were still able to perform sound multivariate analyses.

As this was a retrospective study, we had some missing data on the characteristics studied. Also, there was no prospective protocol for standardized CT scanning at predetermined time points and patients had scans at varying time points during their stay in our hospital. Since we analyzed two radiological patterns of SI, clinical symptoms of secondary ischemia without an infarct on CT or MRI were not taken into account. MRI was not performed in the acute phase of SI and was performed only in a minority of our patients for follow-up of endovascular treatment of the aneurysms. Follow-up by MRI was mainly performed in patients without infarction, and more in SSI than in MSI. Therefore, the fact that MRI is more sensitive is not likely to have biased our results.

Previous literature found that focal or diffuse distribution of vasospasm by TCD or angiogram failed to reliably predict the subsequent pattern of brain infarction [10]. The aim of our study was not to assess the relation between vasospasm and infarction, but to investigate the existence of two different entities of SI. We defined SI on noncontrast CT or MRI and had no full information on presence or absence of vasospasm in all patients. Though vasospasm may contribute to the development of SI, infarction and vasospasm do not always go hand in hand. Obviously, in observational studies SI is a more important outcome event than the presence of arterial spasm by angiography or TCD [5, 15]. Our data give no information whether generalized vasospasm was more present in patients with MSI, or focal vasospasm in patients with SSI. However, other authors found no differences in occurrence or extent of vasospasm in patients with different single or widespread infarct patterns [10]. Therefore, we feel that this is not an important shortcoming of our study.

In this study the presence of more than two vascular risk factors was associated with development of SI. We could not find previous studies with similar findings. Since the relationship between vascular risk factors and SI was not the primary aim of the current study, no definitive conclusions can be drawn from this finding. Future studies should focus on the identification of additional clinical risk factors of SI and unravel the association between vascular risk factors and SI after SAH. Identification of risk factors may improve our understanding of the pathogenesis of SI and help to tailor preventive treatment to the risk profile of individual patients.

In conclusion, our findings suggest that SSI and MSI do not represent two different disease entities. We found that MSI is related to the same characteristics as SSI but to a larger extent, specifically to presence of multiple vascular risk factors, initial loss of consciousness, larger amounts of intraventricular blood and poor clinical status on admission. Our results underline the importance of clinical risk factors for occurrence and extent of secondary infarction after SAH.

\section{Conflict of interest None.}


Open Access This article is distributed under the terms of the Creative Commons Attribution Noncommercial License which permits any noncommercial use, distribution, and reproduction in any medium, provided the original author(s) and source are credited.

\section{References}

1. (1988) Report of World Federation on Neurological Surgeons committee on a universal subarachnoid hemorrhage grading scale. J Neurosurg 68: 985-986

2. Aralasmak A, Akyuz M, Ozkaynak C, Sindel T, Tuncer R (2009) CT angiography and perfusion imaging in patients with subarachnoid hemorrhage: correlation of vasospasm to perfusion abnormality. Neuroradiology 51:85-93

3. Dankbaar JW, Rijsdijk M, van der Schaaf I, Velthuis BK, Wermer MJ, Rinkel GJ (2009) Relationship between vasospasm, cerebral perfusion, and delayed cerebral ischemia after aneurysmal subarachnoid hemorrhage. Neuroradiology 51:813-819

4. Dreier JP, Major S, Manning A, Woitzik J, Drenckhahn C, Steinbrink J, Tolias C, Oliveira-Ferreira AI, Fabricius M, Hartings JA, Vajkoczy P, Lauritzen M, Dirnagl U, Bohner G, Strong AJ (2009) Cortical spreading ischaemia is a novel process involved in ischaemic damage in patients with aneurysmal subarachnoid haemorrhage. Brain 132:1866-1881

5. Frontera JA, Fernandez A, Schmidt JM, Claassen J, Wartenberg KE, Badjatia N, Connolly ES, Mayer SA (2009) Defining vasospasm after subarachnoid hemorrhage: what is the most clinically relevant definition? Stroke 40:1963-1968

6. Hasan D, Vermeulen M, Wijdicks EFM, Hijdra A, van Gijn J (1989) Management problems in acute hydrocephalus after subarachnoid hemorrhage. Stroke 20:747-753

7. Hijdra A, Brouwers PJAM, Vermeulen M, van Gijn J (1990) Grading the amount of blood on computed tomograms after subarachnoid hemorrhage. Stroke 21:1156-1161
8. Pluta RM, Hansen-Schwartz J, Dreier J, Vajkoczy P, Macdonald RL, Nishizawa S, Kasuya H, Wellman G, Keller E, Zauner A, Dorsch N, Clark J, Ono S, Kiris T, Leroux P, Zhang JH (2009) Cerebral vasospasm following subarachnoid hemorrhage: time for a new world of thought. Neurol Res 31:151-158

9. Rabinstein AA, Friedman JA, Weigand SD, McClelland RL, Fulgham JR, Manno EM, Atkinson JL, Wijdicks EF (2004) Predictors of cerebral infarction in aneurysmal subarachnoid hemorrhage. Stroke 35:1862-1866

10. Rabinstein AA, Weigand S, Atkinson JL, Wijdicks EF (2005) Patterns of cerebral infarction in aneurysmal subarachnoid hemorrhage. Stroke 36:992-997

11. Tatu L, Moulin T, Bogousslavsky J, Duvernoy H (1996) Arterial territories of human brain: brainstem and cerebellum. Neurology 47:1125-1135

12. Tatu L, Moulin T, Bogousslavsky J, Duvernoy H (1998) Arterial territories of the human brain: cerebral hemispheres. Neurology 50:1699-1708

13. Treggiari-Venzi MM, Suter PM, Romand JA (2001) Review of medical prevention of vasospasm after aneurysmal subarachnoid hemorrhage: a problem of neurointensive care. Neurosurgery 48:249-261

14. Vergouwen MD, Vermeulen M, Coert BA, Stroes ES, Roos YB (2008) Microthrombosis after aneurysmal subarachnoid hemorrhage: an additional explanation for delayed cerebral ischemia. J Cereb Blood Flow Metab 28:1761-1770

15. Vergouwen MD, Vermeulen M, van Gijn J, Rinkel GJ, Wijdicks EF, Muizelaar JP, Mendelow AD, Juvela S, Yonas H, Terbrugge KG, Macdonald RL, Diringer MN, Broderick JP, Dreier JP, Roos YB (2010) Definition of delayed cerebral ischemia after aneurysmal subarachnoid hemorrhage as an outcome event in clinical trials and observational studies: proposal of a multidisciplinary research group. Stroke 41:2391-2395 